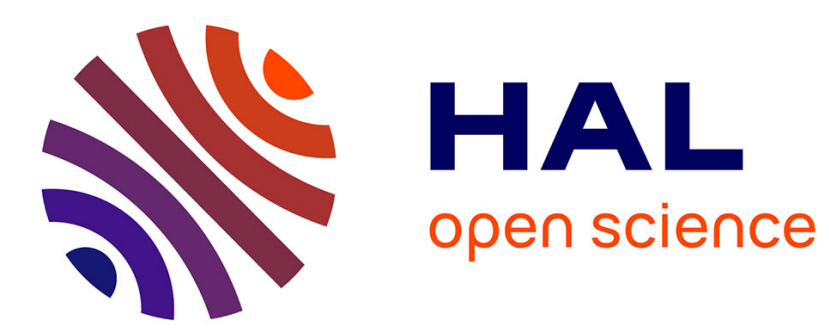

\title{
Acoustic Rotational Manipulation Using Orbital Angular Momentum Transfer
}

Andreas Anhäuser, Régis Wunenburger, Etienne Brasselet

\section{To cite this version:}

Andreas Anhäuser, Régis Wunenburger, Etienne Brasselet. Acoustic Rotational Manipulation Using Orbital Angular Momentum Transfer. Physical Review Letters, 2012, 109 (3), pp.034301. 10.1103/PhysRevLett.109.034301 . hal-00727752

\section{HAL Id: hal-00727752 \\ https://hal.science/hal-00727752}

Submitted on 13 Mar 2018

HAL is a multi-disciplinary open access archive for the deposit and dissemination of scientific research documents, whether they are published or not. The documents may come from teaching and research institutions in France or abroad, or from public or private research centers.
L'archive ouverte pluridisciplinaire HAL, est destinée au dépôt et à la diffusion de documents scientifiques de niveau recherche, publiés ou non, émanant des établissements d'enseignement et de recherche français ou étrangers, des laboratoires publics ou privés. 


\title{
Acoustic Rotational Manipulation Using Orbital Angular Momentum Transfer
}

\author{
Andreas Anhäuser, Régis Wunenburger, ${ }^{*}$ and Etienne Brasselet \\ Université de Bordeaux, LOMA, UMR 5798, F-33400 Talence, France \\ CNRS, LOMA, UMR 5798, F-33400 Talence, France
}

(Received 16 April 2012; published 17 July 2012)

\begin{abstract}
We report on the first quantitative test of acoustic orbital angular momentum transfer to a sound absorbing object immersed in a viscous liquid. This is done by realizing an original experiment that is to spin a millimeter-size target disk using an ultrasonic vortex beam. We demonstrate the balance between the acoustic radiation torque calculated from the Brillouin stress tensor and the viscous torque evaluated from the steady state spinning frequency. Moreover, we unveil a rotational acoustic streaming phenomenon that results from the acoustic angular momentum transfer to the host fluid. We show that it lowers the viscous torque, thereby restoring the torque balance.
\end{abstract}

DOI: 10.1103/PhysRevLett.109.034301

PACS numbers: 43.25.+y, 47.15.G-

Light and sound are known to have mechanical effects on matter that are related to two of their fundamental properties, namely their linear and angular momentum [1]. On the one hand, the transfer of the linear momentum of waves to matter results in a radiation force that enables us to control the position of objects and possibly deform them. On the other hand, the transfer of angular momentum results in a radiation torque that can be used to rotate material systems $[4,5]$.

In optics, these two kinds of mechanical effects have been thoroughly studied from the theoretical, experimental, and applicative point of view. This has led to mature technologies such as optical trapping, tweezing, sorting, chromatography, and rheology [6]. In contrast, in acoustics, the transfer of angular momentum from sound to matter is still in its inception. Nevertheless, the transfer of linear momentum has been extensively studied and is now routinely used, for instance, to manipulate objects or fractionate suspensions.

The identification of acoustic orbital angular momentum (OAM) flux associated to acoustic vortex (AV) beams that bear on-axis phase singularity of the screw-dislocation type started in the 1990s. Such a singularity is locally described by a phase dependence of the pressure field of the form $\exp (i \ell \phi)$, where $\ell$ is an integer called the topological charge [7], and $\phi$ is the azimuthal angle. Since then, several works were dedicated to the production of AVs [7-11], their description [2,12], and the study of their propagation properties $[2,9,13]$. A few experiments that demonstrate acoustic OAM transfer from AVs to matter have been reported, which exploit either the finite angular deviation of absorbing torsional pendulums immersed in air [14-16] or the transient angular deviation of an absorbing fisk immersed in water [17]. Only recently, several theoretical contributions that aim at describing acoustic OAM absorption have been reported [18-21], though restricted to the case of objects immersed in inviscid fluids [22].

Up to now the practical use of acoustic OAM transfer to matter, among them contactless rheology, remain only speculative [21] despite a significant application potential that is related to the very nature of acoustic waves that makes the comparison to optical fields relevant. Indeed, for a given power $\mathcal{P}$, the radiation torque experienced by an absorbing target scales as $\mathcal{P} / \omega$, where $\omega$ is the angular frequency of the wave [19]. This leads to ultrasonic radiation torques that are typically $10^{9}$ times larger than optical ones given their respective typical frequencies $10^{6}$ and $10^{15} \mathrm{~Hz}$. Moreover, considering the generic situation of angular momentum transfer by absorption, the ratio between the heat power deposited in a target and the radiation torque scales as $\omega$. For a given radiation torque, the use of sound instead of light therefore reduces heating by a typical factor of $10^{9}$. Such assets of acoustic waves clearly motivate the development of rotational manipulation of matter based on acoustic OAM. Importantly, a quantitative experimental test of existing predictions of acoustic OAM transfer to matter, which would be of a fundamental interest, is still missing.

In this Letter, we report on the first quantitative test of acoustic OAM transfer to a sound absorbing object immersed in a Newtonian viscous liquid. This is done by realizing an original experiment that is to spin a millimeter-size disk using an AV. The steady spinning frequency is used to analyze the balance of the radiation torque exerted by the AV beam and of the viscous torque exerted by the host fluid.

Setup.-As illustrated in Fig. 1, a disk (D) made of soundabsorbing material (Aptflex 28 from Precision Acoustics), with diameter $2 R=3.15 \mathrm{~mm}$ and thickness $e=0.51 \mathrm{~mm}$, is positioned at the interface between two immiscible liquids, namely a glycerol aqueous solution (fluid 1) and silicone oil (fluid 2), whose characteristics relevant for our experiment are summarized in Table I. The disk is irradiated from below by an AV beam produced by an immersed, spherical, piezoelectric transducer with radius of curvature $F=38.4 \mathrm{~mm}$, diameter $2 a=38 \mathrm{~mm}$, central frequency $f_{\mathrm{ac}}=\omega /(2 \pi)=2.25 \mathrm{MHz}$, and bandwidth $600 \mathrm{kHz}$. In the same spirit as in [14-16], its axisymmetric active surface 
(a)

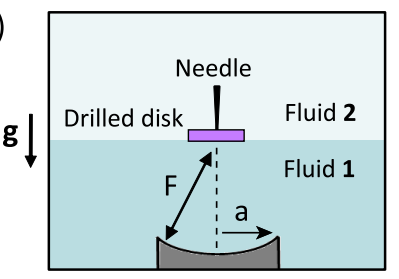

Ultrasonic vortex transducer (b)

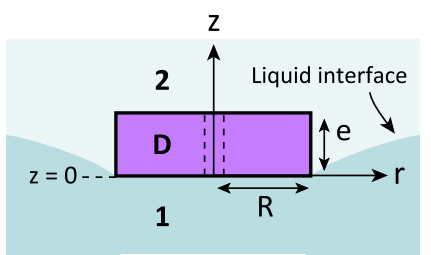

(c)

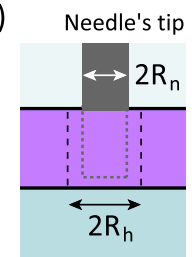

FIG. 1 (color online). (a) Sketch of the experimental setup. (b) Zoom on the acoustic target, a sound absorbing millimeter-size disk. (c) Zoom on the central part of the disk where the needle is inserted into the hole (see text for details).

is equally divided into eight sectors that are independently supplied in parallel by a source that periodically emits sinusoidal wave trains of 200 cycles duration at carrier frequency $f_{\text {ac }}, 1.5 \mathrm{kHz}$ repetition rate, and voltage amplitude $U$. An $\ell=1 \mathrm{AV}$ is produced by using eight different electrical phase delay and impedance-matching quadrupoles inserted between the source and each sector that ensures an $\left(8 f_{\text {ac }}\right)^{-1}$ time delay increase between two adjacent sectors while the radiated acoustic power is the same for all sectors. Hereafter, $(r, \phi, z)$ are cylindrical coordinates where the transducer symmetry axis defines the $z$ axis, the altitude $z=0$ being located at the transducer focal plane, see Fig. 1 .

The characterization of the AV beam is done by using an $85 \mu \mathrm{m}$ diameter active element hydrophone. The measured magnitude and phase of the harmonic acoustic pressure field in the focal plane along the $x$ axis (i.e., at $z=0$ and $\phi=0$ ), are displayed in Figs. 2(a) and 2(b) [see markers] versus the reduced coordinate $k_{1} x, k_{1}=\omega / c_{1}$ being the wave number and $c_{1}$ the sound speed in fluid 1 . The obtained doughnut-shaped field profile is checked to be axisymmetric. This is shown in Fig. 2(c) that displays the pressure magnitude along the circle $C$ of maximal magnitude found to be located at $r=0.55 \mathrm{~mm}$ in the focal plane, which corresponds to $k_{1} x \simeq \pm 5$ in Fig. 2(a). Moreover, the single charge phase singularity is clearly identified from the phase measurement along $C$, see markers on Fig. 2(d), where the solid line refers to the expected behavior $\arg \left(p_{\max }\right)=\phi$.

These experimental data are accurately described by a paraxial modeling [24,25] for the acoustic pressure field produced by our eight-element transducer under assumption of linear, nondissipative propagation, see solid curves in Fig. 2. It turns out that a Laguerre-Gauss (LG) beam with azimuthal index $\ell=1$ and radial index $n=0$ [26], whose approximate expression of the acoustic pressure $p_{\mathrm{LG}}$ close to the focal plane (i.e., for $|z| \ll z_{0}$ with $z_{0}=k_{1} w_{0}^{2} / 2$ ) is

$$
p_{\mathrm{LG}}(r, \phi, z, t)=p_{0}\left(r / w_{0}\right) e^{-r^{2} / w_{0}^{2}+i\left(k_{1} z-\omega t+\phi\right)},
$$

accurately fits both the magnitude and phase of the acoustic pressure field measured in the focal plane over the whole disk surface (i.e., $r<R$ ), see dashed curves in Fig. 2, provided that $w_{0}=1.025 c_{1} / f_{\mathrm{ac}}$ and $p_{0}=\sqrt{2} \exp (1 / 2) p_{\max }$, where $p_{\max }$ is the acoustic pressure magnitude measured along $C$, with $p_{\max } / U=29.2 \mathrm{kPa} / \mathrm{V}$. The amount of incident beam power intercepted by the disk is estimated to be more than $99 \%$ of the total power $\mathcal{P}$ of our LG beam approximation. According to [7], the total acoustic OAM flux along the $z$ axis of the incident $\mathrm{AV}$ beam is $\mathbf{L}_{i}=$ $(\ell \mathcal{P} / \omega) \mathbf{e}_{z}$, with $\mathbf{e}_{z}$ the unit vector along the $z$ axis and $\ell=1$ here.

Acoustic rotation experiment.-As sketched in Fig. 1(b), the meniscus is always found to be pinned at the bottom edge of the disk. As a consequence, both the lateral and top surface of the disk are always in contact with fluid 2 . Moreover, the disk always remains within the beam focal zone of axial extension $2 z_{0}=5.4 \mathrm{~mm}$ within the investigated acoustic power range. This results from the balance of the upward axial acoustic radiation force due to the partial absorption of the linear momentum carried by the incident AV beam, of the interfacial force exerted on the disk by the meniscus and of buoyancy. In addition, the center of the disk is kept along the beam axis owing to a fixed needle that coincides with the $z$ axis and ends into an axial hole drilled through the disk, see Fig. 1(c).

Upon starting the AV emission, the disk slightly rises and starts to spin around the $z$ axis, and finally spins at fixed altitude and frequency, $f_{\text {disk }}$. The rotation frequency depends on the $\mathrm{AV}$ power, as shown in Fig. 3(a) where $f_{\text {disk }}$ is plotted versus $U^{2}$ in log-log scales, which unambiguously demonstrates the scaling of $f_{\text {disk }}$ as $U^{2}$ over more than one decade, see linear fit (solid line). This is expected since $f_{\text {disk }}$ is determined from the balance between (i) the acoustic radiation torque, $\boldsymbol{\Gamma}_{\text {rad }}$, that results from the partially absorbed AV and (ii) the viscous torque, $\boldsymbol{\Gamma}_{\text {visc }}$, exerted by the fluid surrounding the disk. Indeed, since $\Gamma_{\text {rad }} \propto \mathcal{P} \propto$ $U^{2}$ [7] and $\Gamma_{\text {visc }} \propto f_{\text {disk }}$ for creeping flows, we get $f_{\text {disk }} \propto$ $U^{2}$. Towards a quantitative analysis, next we accurately evaluate both $\boldsymbol{\Gamma}_{\text {rad }}$ and $\boldsymbol{\Gamma}_{\text {visc }}$.

TABLE I. Density $\rho$, sound speed $c$, and viscosity $\eta$ of the materials involved in the experiment.

\begin{tabular}{lccc}
\hline \hline & $\begin{array}{c}\text { Aqueous glycerol } \\
\text { solution 79 wt.\% } \\
\text { (Fluid 1) }\end{array}$ & $\begin{array}{c}\text { Silicone oil } \\
1 \mathrm{cSt} \\
\text { (Fluid 2) }\end{array}$ & $\begin{array}{c}\text { Aptflex 28 } \\
\text { (Disk) }\end{array}$ \\
\hline$\rho\left(\mathrm{kg} \cdot \mathrm{m}^{-3}\right)$ & 1204 & 856 & 1020 \\
$c\left(\mathrm{~m} \cdot \mathrm{s}^{-1}\right)$ & 1860 & 913 & 1456 \\
$\eta\left(10^{-3} \mathrm{~Pa} \cdot \mathrm{s}\right)$ & 48 & 0.86 & \\
\hline \hline
\end{tabular}



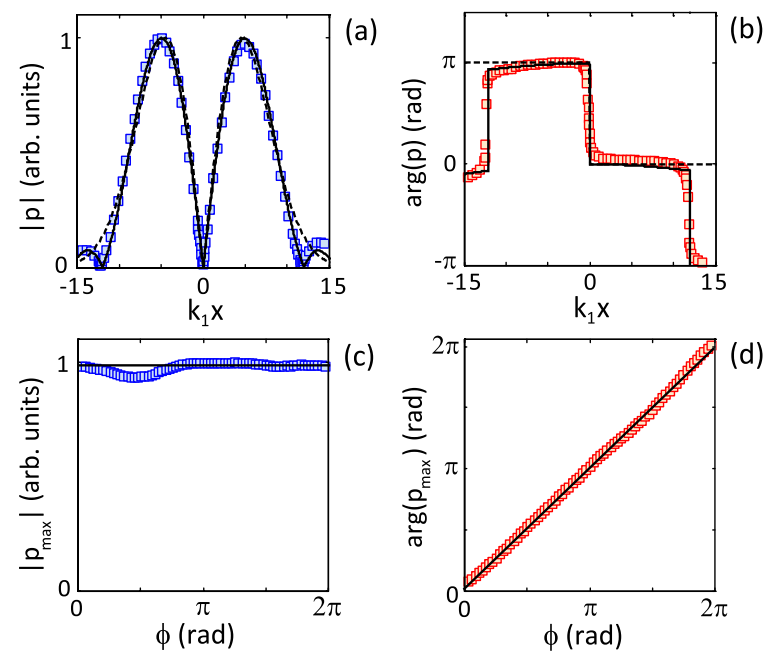

FIG. 2 (color online). (a) Magnitude and (b) phase of the acoustic pressure. Markers: experimental data; solid curves: paraxial model simulations; dashed curves: Laguerre-Gauss approximation of the acoustic vortex beam given by Eq. (1). The disk radius $R$ corresponds to $k_{1} R=12$. (c) Dimensionless magnitude and (d) phase of the acoustic pressure measured along the circle $C$ of maximal magnitude (markers) in the focal plane corresponds to $k_{1} x \simeq \pm 5$, the solid curves being the expected values from the Laguerre-Gauss beam approximation.

Model.-According to Refs. [18,19,27], the radiation torque, i.e., the time-averaged mechanical torque exerted on the irradiated disk by the surrounding fluid considered as perfect, has the following expression, which is valid to second order in the wave amplitude:

$$
\boldsymbol{\Gamma}_{\mathrm{rad}}=\int_{S_{D}} \mathbf{r} \times(-\langle p\rangle \rrbracket-\rho\langle\mathbf{u} \otimes \mathbf{u}\rangle) \cdot d \mathbf{S},
$$

where $S_{D}$ is the disk surface, $\mathbf{r}$ the position vector, $d \mathbf{S}$ the outward pointing surface element vector, $p$ being the fluid acoustic pressure, $\rho$ its density at rest, and $\mathbf{u}$ the acoustic

(a)
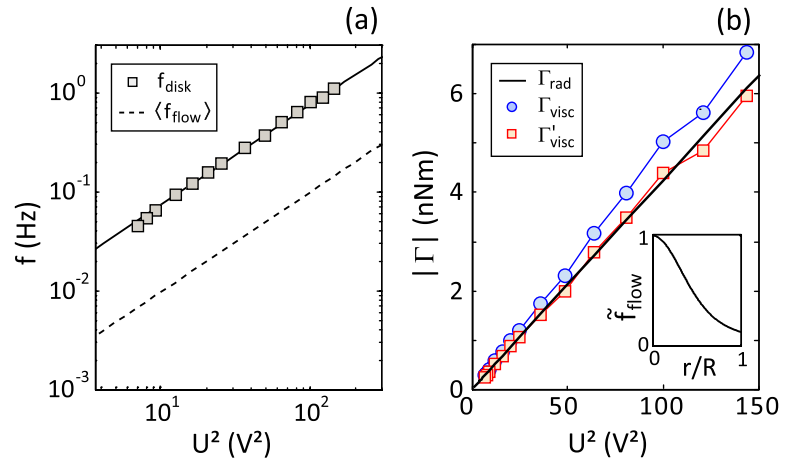

FIG. 3 (color online). (a) Spinning disk frequency $f_{\text {disk }}$ (markers) and predicted average rotation frequency of the (partially soundabsorbing) fluid $1\left\langle f_{\text {flow }}\right\rangle$ (see text for details) versus $U^{2}$. The solid line is the best linear fit to the experimental data. (b) Magnitudes of the radiation torque $\Gamma_{\text {rad }}$ evaluated using Eq. (2) (solid curve), of the viscous torque $\Gamma_{\text {visc }}$ evaluated from $f_{\text {disk }}$ measurements using Eq. (7) (circles), and of the viscous torque $\Gamma_{\text {visc }}^{\prime}$ taking into account the fluid rotation using Eq. (10) (squares). velocity related to $p$ through Euler equation $\rho \frac{\partial \mathbf{u}}{\partial t}=-\nabla p$, $\square$ denoting the identity tensor, $\langle\cdot\rangle$ the time average, and $\otimes$ the dyadic product. Since $S_{D}$ is axisymmetric and $\langle p\rangle=$ $\frac{1}{2} \rho\left\langle u^{2}\right\rangle-\left(2 \rho c^{2}\right)^{-1}\left\langle p^{2}\right\rangle$ does not depend on $\phi$ along $S_{\text {disk }}$, $\int_{S_{D}} \mathbf{r} \times\langle p\rangle d \mathbf{S}=\mathbf{0}$. For the same symmetry reason, $\boldsymbol{\Gamma}_{\text {rad }}$ is axial and can be written as $\boldsymbol{\Gamma}_{\text {rad }}=-\int_{S_{D}} \rho r\left\langle u_{\phi} \mathbf{u} \cdot d \mathbf{S}\right\rangle \mathbf{e}_{z}$. Its quantitative determination obviously requires the knowledge of the acoustic field around the disk.

For this purpose, we assume the propagation through the disk to obey ray acoustics and we neglect refraction at the liquid-liquid interface. In addition, we, respectively, label by $i=1,2, D$, the density $\rho_{i}$, the sound speed $c_{i}$, the acoustic impedance $Z_{i}=\rho_{i} c_{i}$, the wave number $k_{i}=\omega / c_{i}$, and the acoustic attenuation $\alpha_{i}$ of fluid 1, fluid 2, and disk $D$. The pressure field of the AV beam reflected in fluid $1\left(p_{r}\right)$ and of the AV beam transmitted in fluid $2\left(p_{t}\right)$ thus express as

$p_{r}(r<R, \phi, z<0, t)=r_{\text {tot }} p_{0}\left(r / w_{0}\right) e^{\left(-r^{2} / w_{0}^{2}\right)+i\left(-k_{1} z-\omega t+\phi\right)}$,

$p_{t}(r<R, \phi, z>e, t)=t_{\mathrm{tot}} p_{0}\left(r / w_{0}\right) e^{\left(-r^{2} / w_{0}^{2}\right)+i\left(k_{2} z-\omega t+\phi\right)}$,

$p_{t}(r>R, \phi, z>0, t)=t_{12} p_{0}\left(r / w_{0}\right) e^{\left(-r^{2} / w_{0}^{2}\right)+i\left(k_{2} z-\omega t+\phi\right)}$,

where $r_{\text {tot }}=r_{1 D}+t_{1 D} r_{D 2} t_{D 1} X^{2} /\left(1-r_{D 1} r_{D 2} X^{2}\right)$ and $t_{\text {tot }}=$ $t_{1 D} t_{D 2} X /\left(1-r_{D 1} r_{D 2} X^{2}\right)$ with $r_{i j}=\left(Z_{j}-Z_{i}\right) /\left(Z_{j}+Z_{i}\right)$ and $t_{i j}=2 Z_{j} /\left(Z_{j}+Z_{i}\right)$ being, respectively, the pressure reflection and transmission coefficients at normal incidence between media $i$ and $j$, and $X=\exp \left(i k_{D} e-\alpha_{D} e\right)$. Note that $p_{r}$ and $p_{t}$ only weakly depend on $f_{\text {ac }}$, due to the large absorption in the disk $\left(\alpha_{D}=764 \mathrm{~m}^{-1}\right)$ and to the small absolute values of the reflection or transmission coefficients.

Using Eqs. (1) and (3)-(5), we obtain the following expression for $\boldsymbol{\Gamma}_{\mathrm{rad}}$

$$
\boldsymbol{\Gamma}_{\text {rad }}=(\ell / \omega)(1-\mathcal{R}-\mathcal{T}) \mathcal{P} \mathbf{e}_{z}=(\ell / \omega) \mathcal{P}_{\mathrm{abs}} \mathbf{e}_{z}
$$

where $\ell=1$ here, $\mathcal{R}=\left|r_{\text {tot }}\right|^{2}$ and $\mathcal{T}=\left|t_{\text {tot }}\right|^{2} Z_{1} / Z_{2}$ are, respectively, the energy reflection and transmission coefficients, and $\mathcal{P}_{\text {abs }}$ is the power absorbed by the disk [28]. The calculated behavior of $\Gamma_{\text {rad }}$ versus $U^{2}$ is plotted in Fig. 3(b) (solid curve). Interestingly, we stress that Eq. (6) can be rewritten in order to emphasize the acoustic OAM flux balance. Namely, $\boldsymbol{\Gamma}_{\text {rad }}=\mathbf{L}_{i}-\mathbf{L}_{r}-\mathbf{L}_{t}$, where $\mathbf{L}_{i}=$ $\frac{\ell}{\omega} \mathcal{P} \mathbf{e}_{z}$ (respectively, $\mathbf{L}_{r}=\frac{\ell}{\omega} \mathcal{R} \mathcal{P} \mathbf{e}_{z}$ and $\mathbf{L}_{t}=\frac{\ell}{\omega} \mathcal{T} \mathcal{P} \mathbf{e}_{z}$ ) is the total OAM flux along associated to the incident (respectively, reflected and transmitted) AV.

On the other hand, $\boldsymbol{\Gamma}_{\text {visc }}$ can be accurately evaluated by taking into account that fluid 1, which is 56 times more viscous than fluid 2, is in contact with the circular bottom of the disk only, and that the Reynolds number $\operatorname{Re}_{1}=\Omega R^{2} \rho_{1} / \eta_{1}$ associated with the flow of fluid 1 is 
smaller than 0.2 in our experiment; i.e., the hypothesis of creeping flow in fluid 1 is valid [29]. Therefore, we consider the viscous torque to be half the one experienced by an infinitely thin disk in the creeping flow limit [31]:

$$
\boldsymbol{\Gamma}_{\mathrm{visc}}=-(16 / 3) \eta_{1} R^{3} \Omega \mathbf{e}_{z}
$$

where $\Omega=2 \pi f_{\text {disk }}$ is the steady angular frequency of the spinning disk [32]. The dependence of $\Gamma_{\text {visc }}$ on $U^{2}$ is therefore evaluated from the measured values of $f_{\text {disk }}$, see Fig. 3(b) (circles).

The steady rotation of the disk implies the stationarity of its angular momentum, hence $\boldsymbol{\Gamma}_{\text {rad }}+\boldsymbol{\Gamma}_{\text {visc }}=\mathbf{0}$. However, from Fig. 3 we find that $\left|\Gamma_{\text {visc }}\right|$ is typically $15 \%$ larger than $\left|\Gamma_{\text {rad }}\right|$ over the whole investigated range of input AV beam power. Hereafter, we argue that such an observation has a physical meaning and an attempt to describe it is presented.

Acoustic rotational streaming.-Although acoustic propagation in fluids has been up to now considered as nondissipative, dissipation actually occurs as an unavoidable consequence of viscosity and heat conduction [33]. Acoustic attenuation is well known to trigger an axial steady flow usually called acoustic streaming due to the transfer of linear momentum from a progressive acoustic wave to a sound absorbing fluid [34]. In the present experiment, the $\mathrm{AV}$ attenuation by the fluids themselves is therefore expected to result not only in usual acoustic axial streaming but also in its rotational counterpart called hereafter "acoustic rotational streaming," triggered by the radiation torque exerted on the fluid bulk as a result of acoustic OAM transfer to it. Such an acoustic rotational streaming can indeed be observed with naked eyes in the vicinity of the beam focus in absence of the disk by looking at moving dust particles lying on the liquid interface. Since the fluid and the disk are corotating, we expect the fluid flow to lower the viscous torque exerted by the fluid on the disk. This issue is addressed hereafter.

The structure and magnitude of the steady velocity field, $V(r, \phi, z)$, of this rotational streaming flow can be predicted in the beam focal zone. This is done by noting that the latter, incompressible, small Reynolds number flow mainly develops in the focal zone, where the bulk acoustic OAM transfer is the largest, and that the bottom of the disk constitutes a stagnation surface for this flow. Consequently, we consider $V_{z}$, hence $V_{r}$ (which is of the same magnitude as $V_{z}$ as a consequence of mass conservation), as negligible in the focal zone. Moreover, we assume the purely rotational flow to be $z$ invariant in the focal zone. As a consequence, $\mathbf{V}=V_{\phi}(r) \mathbf{e}_{\phi}$ satisfies the following form of the Stokes equation:

$$
\frac{\eta}{r^{2}} \frac{\partial}{\partial r}\left[r^{3} \frac{\partial}{\partial r}\left(\frac{V_{\phi}}{r}\right)\right]=\frac{\gamma}{r}
$$

where $\gamma$ is the radiation torque density exerted on the fluid along $z$. Since the axial acoustic OAM flux density along the $z$ axis, $M_{z z}$, satisfies $\left\langle M_{z z}\right\rangle=\ell I / \omega$ where $I=\left\langle p u_{z}\right\rangle$ is the time-averaged axial acoustic energy flux density [19], the balance of angular momentum for an axisymmetric fluid volume bounded by radii $r$ and $r+d r$ and altitudes $z$ and $z+d z$ between times $t$ and $t+d t$ gives $\gamma=\alpha \ell I / \omega$ where $\alpha$ is the acoustic attenuation coefficient of the fluid. The solution of Eq. (8) in fluid 1 can therefore be calculated using Eq. (1). One finds

$$
V_{\phi}(r)=\frac{3 \alpha_{1} p_{0}^{2} w_{0}^{2}}{32 \omega \eta_{1} \rho_{1} c_{1}} \frac{1-\exp \left[-2 r^{2} / w_{0}^{2}\right]}{r} .
$$

The corresponding fluid rotation frequency $f_{\text {flow }}(r)=$ $(2 \pi)^{-1} V_{\phi}(r) / r$ exhibits a strong $r$ dependence, as shown in the inset of Fig. 3(b) where $\tilde{f}_{\text {flow }}=f_{\text {flow }}(r) / f_{\text {flow }}(0)$ is plotted versus $r / R$. In order to take account of the influence of the inhomogeneous fluid rotation on the viscous torque exerted on the spinning disk, we consider the disk to experience a modified viscous torque $\Gamma_{\text {visc }}^{\prime}=$ $\int_{0}^{R} d \boldsymbol{\Gamma}_{\text {visc }}^{\prime}$, where $d \boldsymbol{\Gamma}_{\text {visc }}^{\prime}$, defined as the elementary viscous torque exerted on an axisymmetric disk surface element bounded by radii $r$ and $r+d r$ by the fluid located between $r$ and $r+d r$ and rotating at angular frequency $\Omega_{\text {flow }}(r)=2 \pi f_{\text {flow }}(r)$, is assumed to be proportional to $\Omega-\Omega_{\text {flow }}(r)$. Under this assumption,

$$
\Gamma_{\mathrm{visc}}^{\prime}=(16 / 3) \eta_{1} R^{3}\left(\Omega-\left\langle\Omega_{\text {flow }}\right\rangle\right) \mathbf{e}_{z},
$$

where $\left\langle\Omega_{\text {flow }}\right\rangle=\left(3 / R^{3}\right) \int_{0}^{R} \Omega_{\text {flow }}(r) r^{2} d r$. The best agreement between $\left|\Gamma_{\text {rad }}\right|$ and $\left|\Gamma_{\text {visc }}^{\prime}\right|$ [see square symbols in Fig. 3(b)] is found with $\alpha_{1}=18 \mathrm{~m}^{-1}$. This value can be hardly compared with data from literature, which are missing for the actual water-glycerol mixture used in our experiment at $f_{\mathrm{ac}}=2.25 \mathrm{MHz}$ and temperature $25^{\circ} \mathrm{C}$. Nevertheless, we checked that the order of magnitude of the latter value is consistent with the available data obtained in experimental conditions that are the closest to ours [35,36]. Consequently, this flow model supports our claim that the acoustic rotational streaming of the host fluid, whose average rotation frequency $\left\langle f_{\text {flow }}\right\rangle=$ $\left\langle\Omega_{\text {flow }}\right\rangle /(2 \pi)$ versus $U^{2}$ is plotted in Fig. 3(a) (dashed curve), does influence the spinning disk frequency.

We believe that these results have a broad application potential. While acoustic OAM transfer to highly absorbing targets could be applied to contactless, in situ rheology, in a similar manner to optical microrheology [37,38], acoustic rotational streaming could be originally applied to the in situ measurement of the acoustic attenuation of fluids by using weakly absorbing and/or highly reflecting targets. Moreover, acoustic OAM transfer could be advantageously used to trigger the rotation of trapped objects. Finally, acoustic rotational streaming appears as a new tool for the local actuation of fluids and soft solids, thus opening new perspectives in acoustic microfluidics [39] and biomedical ultrasonic imaging. 
The authors thank $\mathrm{Ph}$. Maire for the realization of the electronic delay circuit. This work was partly supported by Conseil Régional d'Aquitaine.

*Corresponding author. r.wunenburger@loma.u-bordeaux1.fr

[1] Actually the linear and angular pseudomomentum in the case of acoustic waves [2,3].

[2] J.-L. Thomas and R. Marchiano, Phys. Rev. Lett. 91, 244302 (2003).

[3] M.E. McIntyre, J. Fluid Mech. 106, 331 (1981).

[4] M. E. J. Friese, J. Enger, H. Rubinsztein-Dunlop, and N. R. Heckenberg, Phys. Rev. A 54, 1593 (1996).

[5] M. E. J. Friese, T. A. Nieminen, N. R. Heckenberg, and H. Rubinsztein-Dunlop, Nature (London) 394, 348 (1998).

[6] D. G. Grier, Nature (London) 424, 810 (2003).

[7] B. T. Hefner and P. L. Marston, J. Acoust. Soc. Am. 106, 3313 (1999).

[8] S. Gspan, A. Meyer, S. Bernet, and M. Ritsch-Marte, J. Acoust. Soc. Am. 115, 1142 (2004).

[9] R. Marchiano and J.-L. Thomas, Phys. Rev. E 71, 066616 (2005).

[10] P.Z. Dashti, F. Alhassen, and H. P. Lee, Phys. Rev. Lett. 96, 043604 (2006).

[11] J. L. Ealo, J. C. Prieto, and F. Seco, IEEE Trans. Ultrason. Ferroelectr. Freq. Control 58, 1651 (2011).

[12] J. Lekner, J. Acoust. Soc. Am. 120, 3475 (2006).

[13] P. L. Marston, J. Acoust. Soc. Am. 124, 2905 (2008).

[14] K. Volke-Sepulveda, A. O. Santillan, and R. R. Boullosa, Phys. Rev. Lett. 100, 024302 (2008).

[15] K. D. Skeldon, C. Wilson, M. Edgar, and M. J. Padgett, New J. Phys. 10, 013018 (2008).

[16] C. Wilson and M.J. Padgett, New J. Phys. 12, 023018 (2010).

[17] C. E. M. Demore, Z. Yang, A. Volovick, S. Cochran, M. P. MacDonald, and G. C. Spalding, Phys. Rev. Lett. 108, 194301 (2012).

[18] L. Zhang and P. L. Marston, J. Acoust. Soc. Am. 129, 1679 (2011).

[19] L. Zhang and P.L. Marston, Phys. Rev. E 84, 065601 (2011).

[20] F. G. Mitri, T. P. Lobo, and G. T. Silva, Phys. Rev. E 85, 026602 (2012).

[21] G. T. Silva, T. P. Lobo, and F. G. Mitri, Europhys. Lett. 97, 54003 (2012).

[22] Nevertheless, note that a way to take into account the effect of viscous boundary layers has been suggested in $[19,20]$ in the context of Bessel vortices. This might be done by extending the work of [23] originally performed for orthogonal plane waves to AV beams.

[23] F. H. Busse and T. G. Wang, J. Acoust. Soc. Am. 69, 1634 (1981).

[24] G.S. Kino, in Acoustic Waves: Devices, Imaging and Analog Signal Processing, Prentice-Hall Signal Processing Series edited by A. V. Oppenheim (PrenticeHall, Englewood Cliffs, NJ, 1987).

[25] The use of a paraxial model is justified by the moderate aperture angle of the transducer $\theta_{0}=\arcsin (a / F)=30^{\circ}$.

[26] L. Allen, M. W. Beijersbergen, R. J. C. Spreeuw, and J. P. Woerdman, Phys. Rev. A 45, 8185 (1992).

[27] G. Maidanik, J. Acoust. Soc. Am. 30, 620 (1958).

[28] Actually, we took into account the presence of the disk hole by correcting $\mathcal{P}_{\text {abs }}$ with a multiplying factor that has been calculated to be 0.97 .

[29] According to [30], the magnitude of finite Reynolds number corrections to Eq. (7) is predicted to be less than $1 \%$ for $\operatorname{Re}_{1}<4$.

[30] Y. G. Ovseenko, Mekh. Zhidk. Gaza 4, 144 (1969).

[31] V. G. Levich, Physicochemical Hydrodynamics (PrenticeHall, Englewood Cliffs, NJ, 1962).

[32] Eq. (7) slightly underestimates the actual viscous torque exerted on the disk since it takes into account neither the viscous torque exerted by fluid 2 along the top and the circumference of the disk, which is estimated to be approximately $3 \%$ of $\Gamma_{\text {visc }}$ by using an ellipsoidal fit to the cylindrical disk and the predictions of Ref. [30], nor the reduction of the viscous torque due to the axial hole, which is estimated to be less than $1 \%$ of $\Gamma_{\text {visc }}$, nor the viscous torque $\boldsymbol{\Gamma}_{\text {gap }}$ associated to the rotation of the disk around the needle, whose magnitude is estimated to be of the order of $0.1 \%$ of $\Gamma_{\text {visc }}$ using a model of $z$ invariant creeping flow in the gap which predicts $\boldsymbol{\Gamma}_{\text {gap }}=$ $-2 \pi \eta_{2} e R_{h}^{2} / \ln \left(R_{h} / R_{n}\right) \Omega \mathbf{e}_{z}$, where $R_{n}=0.24 \mathrm{~mm}$ (respectively, $R_{h}=0.32 \mathrm{~mm}$ ) is the needle (respectively, hole) radius [Fig. 1(c)].

[33] P. M. Morse and K. U. Ingard, Theoretical Acoustics (Princeton University, Princeton, NJ, 1968).

[34] Nonlinear Acoustics, edited by M. F. Hamilton and D. T. Blackstock (Academic, New York, 1997).

[35] G. S. Darbari, R.P. Singh, and G.S. Verma, Nuovo Cimento B 41, 15 (1966).

[36] G. Madelin, D. Gricker, J.-M. Franconi, and E. Thiaudiere, Ultrasonics 44, 272 (2006).

[37] N. R. H. A. I. Bishop, T. A. Nieminen, and H. RubinszteinDunlop, Phys. Rev. Lett. 92198104 (2004).

[38] V. J. Y. T. N. Murazawa, S. Juodkazis, and H. Misawa, Europhys. Lett. 73, 800 (2006).

[39] J. Friend and L. Y. Yeo, Rev. Mod. Phys. 83, 647 (2011). 